\title{
Privacy Preserving Data Mining Research: Current Status and Key Issues
}

\author{
Xiaodan $\mathrm{Wu}^{1}$, Chao-Hsien $\mathrm{Chu}^{2}$, Yunfeng Wang ${ }^{1}$, Fengli Liu ${ }^{1}$, and Dianmin Yue ${ }^{1}$ \\ ${ }^{1}$ School of Management, Hebei University of Technology, Tianjin 300130, China \\ xwu@hebut.edu.cn, ywang@hebut.edu.cn, liufengli312@163.com, \\ dyue@hebut.edu.cn \\ ${ }^{2}$ College of Information Sciences and Technology, The Pennsylvania State University, 301K \\ IST Building, University Park, PA 16802, USA \\ chulist.psu.edu
}

\begin{abstract}
Recent advances in the Internet, in data mining, and in security technologies have gave rise to a new stream of research, known as privacy preserving data mining (PPDM). PPDM technologies allow us to extract relevant knowledge from a large amount of data, while hide sensitive data or information from disclosure. Several research questions have often being asked: (1) what kind of option available for privacy preserving? (2) Which method is more popular? (3) how to measure the performance of these algorithms? And (4) how effective of these algorithms in preserving privacy? To help answer these questions, we conduct an extensive review of 29 recent references from years 2000 to 2006 for analysis.
\end{abstract}

Keywords: Privacy preserving; data mining.

\section{Introduction}

Data mining is a well-known technique for automatically and intelligently extracting information or knowledge from a large amount of data, which, however, can also disclosure sensitive information about individuals compromising the individual's right to privacy. Moreover, data mining techniques can reveal critical information about business transactions, compromising the free competition in a business setting [1]. Therefore, privacy preserving data mining (PPDM) has becoming an increasingly important field of study.

PPDM is a new era of research in data mining, where data mining algorithms are analyzed for possible infringement in privacy. PPDM research usually takes one of the three philosophical approaches: (1) data hiding, in which sensitive raw data like identifiers, name, addresses, etc. were altered, blocked, or trimmed out from the original database, in order for the users of the data not to be able to compromise another person's privacy; (2) rule hiding, in which sensitive knowledge extracted from the data mining process be excluded for use, because confidential information may be derived from the released knowledge; and (3) secure multiparty computation, where distributed data are encrypted before released or shared for computations; thus, 
no party knows anything except its own inputs and the results. The ultimate goal of PPDM is to develop efficient algorithms that allow one to extract relevant knowledge from a large amount of data, while prevent sensitive data and information from disclosure or inference.

PPDM is a fast growing research area. Given the number of different algorithms have been developed over the past years, there is an emerging need of synthesizing literature to understand the nature of problems, identify potential research issues, standardize new research area, and evaluate the relative performance of different approaches [1] [28].

\section{Related Work}

Several researchers have attempted to synthesize the literature. Kantarcioglu and Clifton [7] suggested that adopting a common framework for discussing privacy preservation will enable next generation data mining technology to make substantial advances in alleviating privacy concerns. Verykios et al. [28] analyzed the state-ofthe-art, classified the proposed algorithms from five different dimensions: data distribution, data modification, data mining algorithm, data or rule hiding, and privacy preservation. They also suggested a set of metrics for assessing PPDM performance. Bertino et al. [1] proposed a taxonomy for classifying existing PPDM algorithms. They also developed a framework and based upon which to evaluate the relative performance of selected heuristic-based hiding algorithms. In this paper, we propose to consolidate and simplify the taxonomy brought by [1]. We have also attempted to examine the relative performance of PPDM from individual component level instead of the complete PPDM algorithms.

\section{Current Status}

We present a simplified classification scheme, adopted from early studies, to guide the review process. The proposed taxonomy contains four levels (see Figure 1). This scheme differs from existing studies in two ways: (1) we treat data modification methods as part of privacy preserving techniques, and (2) we consider the purpose of hiding as a key classifier and place it before data mining task/algorithm level. The number appears below each category indicated its percentage of usage (popularity).

\subsection{Data Distribution}

The PPDM algorithms can be first divided into two major categories, centralized and distributed data, based on the distribution of data. In a centralized database (C-DB) environment, data are all stored in a single database; while, in a distributed database (D-DB) environment, data are stored in different databases [3]-[6] [13]-[22] [27] [29] [31]. Distributed data scenarios can be further classified into horizontal and vertical data distributions. Horizontal distributions refer to the cases where different records of the same data attributes are resided in different places [7] - [11] [26]; while in a vertical data distribution, different attributes of the same record of data are resided in different places [2] [23] [24] [25] [30] [33]. As can be seen, earlier research has been 


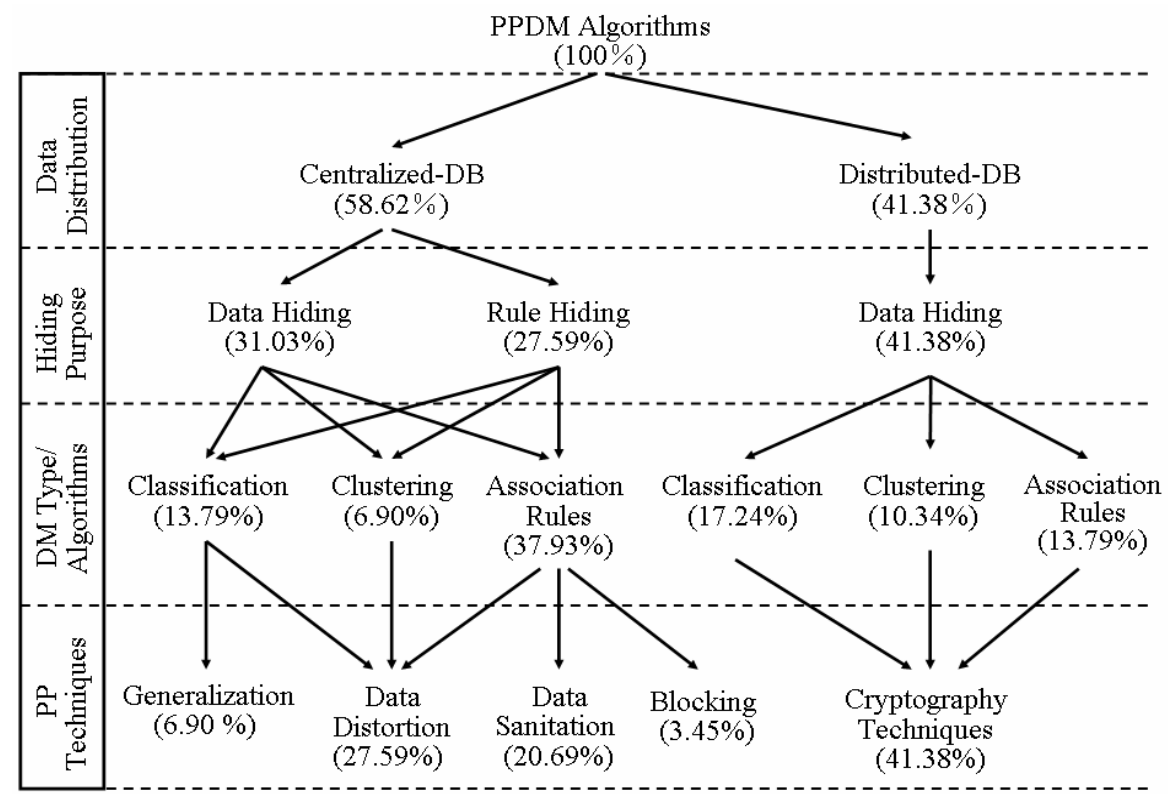

Fig. 1. Taxonomy of PPDM algorithms

predominately focused on dealing with privacy preservation in a centralized DB. The difficulties of applying PPDM algorithms to a distributed DB can be attributed to: first, the data owners have privacy concerns so they may not willing to release their own data for others; second, even if they are willing to share data, the communication cost between the sites is too expensive. However, in today's global digital environment, most data are often stored in different sites, so more attention and research should be focused on distributed PPDM algorithms.

\subsection{Hiding Purposes}

The PPDM algorithms can be further classified into two types, data hiding and rule hiding, according to the purposes of hiding. Data hiding refers to the cases where the sensitive data from original database like identity, name, and address that can be linked, directly or indirectly, to an individual person are hided. In contrast, in rule hiding, we remove the sensitive knowledge (rule) derived from original database after applying data mining algorithms. We summarize the related literature in Table 1. Majority of the PPDM algorithms used data hiding techniques. This is especially true in a distributed database environment, as the techniques can be used to prevent individual information from being discovered by other parties in the joint computational process. Please note that most PPDM algorithms hide sensitive patterns by modifying data. Also, at present, the rule hiding techniques is only being adopted by association rule mining for centralized DB. The reason for such a restriction is mainly due to its ease of implementation. 
Table 1. Summary of the hiding purpose of PPDM

\begin{tabular}{cll}
\hline \multirow{2}{*}{ Purpose } & \multicolumn{1}{c}{ References } \\
\cline { 2 - 3 } Data hiding & \multicolumn{1}{c}{ Centralized DB } & \multicolumn{1}{c}{ Distributed DB } \\
\hline \multirow{2}{*}{ [3] [5] [6] [17] [18] [20][29] } & $[2][7][8][9] 10][11][23][24][25]$ \\
Rule hiding & {$[14][15][16][19][27][21][22]$} & \multicolumn{1}{c}{ [27][30][33] } \\
\hline
\end{tabular}

\subsection{Data Mining Tasks/Algorithms}

Currently, the PPDM algorithms are mainly used on the tasks of classification, association rule and clustering. Association analysis involves the discovery of associated rules, showing attribute value and conditions that occur frequently in a given set of data. Classification is the process of finding a set of models (or functions) that describe and distinguish data classes or concepts, for the purpose of being able to use the model to predict the class of objects whose class label is unknown. Clustering Analysis concerns the problem of decomposing or partitioning a data set (usually multivariate) into groups so that the points in one group are similar to each other and are as different as possible from the points in other groups. We summarize the distribution of literature in Table 2. About 52\% of the PPDM algorithms used association rule method for mining data, followed by classification, and then clustering. Compared with association rule mining, classification rule mining is more complicated to perform. Also, unlike association rules mining, which deals with existing data items, classification deals with attributes and its values. Moreover, instead of associating links between attribute values, it also needs to classify the attributes of each dataset.

Table 2. Summary of PPDM for data mining tasks

\begin{tabular}{lll}
\hline \multicolumn{1}{c}{$\begin{array}{c}\text { Data mining } \\
\text { techniques }\end{array}$} & \multicolumn{1}{c}{ References } \\
\cline { 2 - 3 } Classification & {$[3][6][13][29]$} & \multicolumn{1}{c}{ Centralized DB } \\
Association Rules & {$[4][5][14][15][16][20][21][22][27][31][10][25][30]$} & {$[7][8][25][26]$} \\
Clustering & {$[17][18]$} & {$[9][11][24]$} \\
\hline
\end{tabular}

\subsection{Privacy Preservation Techniques}

We can further divide PPDM algorithms according to privacy preservation techniques used. Four techniques - sanitation, blocking, distort, and generalization -- have been used to hide data items for a centralized data distribution. The idea behind data sanitation is to remove or modify items in a database to reduce the support of some frequently used itemsets such that sensitive patterns cannot be mined. The blocking approach replaces certain attributes of the data with a question mark. In this regard, the minimum support and confidence level will be altered into a minimum interval. As long as the support and/or the confidence of a sensitive rule lie below the middle in these two ranges, the confidentiality of data is expected to be protected. Also 
known as data perturbation or data randomization, data distort protects privacy for individual data records through modification of its original data, in which the original distribution of the data is reconstructed from the randomized data. These techniques aim to design distortion methods after which the true value of any individual record is difficult to ascertain, but "global" properties of the data remain largely unchanged. Generalization transforms and replaces each record value with a corresponding generalized value.

The privacy preservation technique used in a distributed database is mainly based on cryptography techniques. SMC algorithms deal with computing any function on any input, in a distributed network where each participant holds one of the inputs, while ensuring that no more information is revealed to a participant in the computation than can be inferred from that participant's input and output. The distribution of the literature is given in Table 3. Data distort is the most popular method used in hiding data, followed by data sanitation and generalization. If one wants to obtain data mining results from different data sources, then the only method can be used is a cryptography technique. Since the parties who use SMC operators cannot reveal anything from others except final results, it can have benefits of both accuracy of data mining results and the privacy of the database.

Table 3. Summary for privacy preservation techniques

\begin{tabular}{lll}
\hline \multicolumn{2}{c}{ PP Techniques } & \multicolumn{1}{c}{ References } \\
\hline & Data Sanitation & {$[14][15][16][19][22][27]$} \\
C-DB & Blocking & {$[21]$} \\
& Data Distort & {$[3][4][5][6][17][18][20][31]$} \\
& Generalization & {$[13][29]$} \\
D-DB & Cryptography Techniques & {$[2][7][8][9][10][11][23][24][24][26][30][33]$} \\
\hline
\end{tabular}

\subsection{Evaluation Criteria}

The metrics commonly used in early research are:

1. Efficiency, which concerns the ability of an algorithm to execute with good performance in terms of all the resources implied by the algorithm; Performance is assessed, as usually, in terms of computational costs [14] [15] [18] [19] [21] [27], and in case of distributed algorithms, in terms of the communication costs incurred during information exchanges [2] [7] [8] [9] [10] [11] [23] [24] [25] [27] [30] [33].

2. Effectiveness, which considers both the capability of hiding sensitive information [3] [4] [5] [6] [13] [14] [15] [17] [18] [19] [20] [21] [22] [27] [29] [31] and accuracy of the data mining results [3] [4] [5] [6] [13] [14] [4] [16] [17] [18] [19] [20] [21] [27] [29] [31]. For example, the accuracy of data sanitation technique may be measured in terms of hiding failure, that is, the portion of sensitive information that is not hidden by the technique, and misses cost, that occurs when some legitimate patterns are hidden by accident.

3. Scalability, closely related to computational cost, concerns the size of problem the algorithm can solve within a reasonably acceptable timeframe. The higher the computational cost, the less chance the algorithms be used for solving larger size of problems. 


\subsection{Performance Assessment}

Assessing the relative performance of PPDM algorithms is a very difficult task, as it is often the case that no single algorithm outperforms others on all possible criteria. Also, for maximum flexibility, we rate the relative merit of individual module that comprised by the PPDM algorithm. The rating is given in three different levels -high, medium, and low. We summarize the results in Table 4, and discuss the general principles below.

Table 4. Relative performance of PPDM components

\begin{tabular}{|c|c|c|c|c|}
\hline Elements & Computational Cost & Privacy Preserving & Accuracy of Mining & Scalability \\
\hline \multicolumn{5}{|l|}{ Hiding Purpose: } \\
\hline Data Hiding & Low & Contingent & Contingent & High \\
\hline Rule Hiding & High & Contingent & Contingent & Low \\
\hline \multicolumn{5}{|l|}{ Data Mining Tasks: } \\
\hline Classification & Low & N/A & Contingent & High \\
\hline Clustering & High & N/A & Contingent & Low \\
\hline Association Rule & Low & N/A & Contingent & High \\
\hline \multicolumn{5}{|c|}{ Privacy Preserving Technique: } \\
\hline Sanitation & Medium & Medium & Medium & Low \\
\hline Distortion & Low & High & Low & High \\
\hline Blocking & Medium & Low & Medium & Low \\
\hline Generalization & Low & High & Medium & High \\
\hline Cryptography & High & High & High & Low \\
\hline
\end{tabular}

In term of computational efficiency, rule hiding is less efficient than data hiding, because one has to identify the items that contribute to the sensitive rule first and then hide the rule. For the privacy requirement, we think hiding rule is more critical than hiding data, because after the sensitive rules are found, more information is possible to be inferred. This is not to say that rule hiding is more accurate than data hiding. The selection of either hiding data or rule often depends on the goal of privacy preserving (hiding purpose) and data distribution. For instance, we can only hide data under a distributed database environment.

In general, clustering is more complex than classification (including association rules) because it often requires using an unsupervised learning algorithm. The algorithm used for association rule and classification can learn from known results, thus, they are more efficient. However, the preserving power and accuracy are highly dependent on hiding technique used or the algorithm used, not the data mining task. Assessing the performance of traditional data mining algorithms is out of the scope of this study.

The key idea behinds sanitization type of algorithms is to hide the set of frequent patterns from containing highly sensitive knowledge. The complexity of this type of algorithm is $O(n 1 * N \log N)$, where $n 1$ is the number of restrictive patterns and $N$ the number of transactions in the database [14]. Thus, comparing with other methods, the computational cost of sanitation type of algorithm is medium. Study has shown that the more sensitive patterns are hidden, the more legitimate patterns are missed, so it also degrades the accuracy of data mining results. Moreover, the optimal sanitization 
is proved to be NP-hard in the context of association rule mining; therefore, it is computationally intensive as compared with a data perturbation approach.

The inherent mechanism of blocking and sanitization is basically similar. The former uses a '?' notation to replace selected items to be protected, while the latter deletes or modifies these items from viewing; thereby, their complexity are almost the same. However, the privacy preserving capability of blocking is lower than sanitization. Moreover, like sanitization, blocking technique is $N P$-hard. Therefore, these two modification methods cannot be used to solve larger size of problems.

Most existing studies which used distortion method focus on maintaining the level of privacy disclosure and knowledge discovery ability. It seems that efficiency and computational cost are not the issues for distortion method. In general, data distortion algorithms have good effectiveness in hiding data. However, these methods are not without faults. First, the distorting approach only works if one does not need to reconstruct the original data values. Thus, if the data mining task changed, new algorithms need to be developed to reconstruct the distributions. Second, this technique considers each attribute independently; as a result, when the number of attributes became large, the accuracy of data mining results will degrade significantly. Finally, there is a trade-off between accuracy of data mining results and data security using distortion methods. These methods may not be suitable for mining data in situations requiring both high accuracy and high security.

The generalization technique has been widely used in protecting individual privacy with the k-anonymity model in the past; however, it is relatively new to the data mining community. Since generalization has the advantage of not modifying the true value of attributes, it may have higher accuracy of data mining result than data distortion techniques. The complexity of this algorithm is $\mathrm{O}(\mathrm{k} \log \mathrm{k})$, where the constant in the big-O is less than 4. Although the runtime of this algorithm is exponential in $\mathrm{k}$, its efficiency can be greatly enhanced as suggested by [12].

Cryptography-based SMC has the highest accuracy in data mining and good privacy preservation capability as well; however, it has strict usages as it is only applicable to a distributed data environment. Two models of SMC are available: semihonest model and malicious model. The semi-honest models assume each party follows the protocol rules, but is free to later use what it sees during execution to compromise security; while the malicious model assumes parties can arbitrarily "cheat," and such cheating will not compromise either security or the results. How to prevent or detect malicious party in a computation process is an unsolved issue. Not to mention that SMC has the burden of high communicational cost, when the number of parties participated increased. Usually, the communicational cost increases at the exponential speed when data size increases linearly. Also, different problems need different protocols and the complexities vary naturally [30].

\section{Key Issues}

The following issues/directions were derived for future research. First, currently several groups of researchers have devoted effort in studying PPDM from different perspectives (e.g., statistics, database, data mining, knowledge discovery, and 
information security), but they tend to use different terminology to describe similar or related practice. For instance, people have used data modification, data perturbation, data sanitation, data hiding, and preprocessing as possible methods for preserving privacy; however, all are in fact related to the use of some types of technique to modify original data so that private data and knowledge remain private even after the mining process. Lacking a common language for discussions will cause misunderstanding and slow down the research breakthrough. Therefore, there is an emerging need of standardizing the terminology and PPDM practice.

Second, although many machine learning methods have been used for classification, clustering, and other data mining tasks (e.g., diagnose, prediction, optimization), currently only the association rules method has been predominately used. It would be interesting to see how to extend the current technique and practice into other problem domains or data mining tasks. It may be also interesting in using different mining algorithms, especially the nature-based intelligent technologies such as genetic algorithms, neural networks, ant colony, and immune systems to mining and preserving privacy. Furthermore, it is important to find the privacy preserving technique that is independent of data mining task and algorithm so that after applying privacy preserving technique a database can be released without being constrained to the original task and mining algorithms.

Third, most prior PPDM algorithms were developed for use with data stored in a centralized database. However, in today's global digital environment, data is often stored in different sites. With recent advances in information and communication technologies, the distributed PPDM methodology may have a wider application, especially in medical, health care, banking, military and supply chain scenarios. In addition, current PPDM algorithms mainly focus on preserving tuples in the database. Fundamental questions such as where does privacy may happen, what kind of data, data attributes and data type need to preserve remain to be explored.

Fourth, data hiding techniques have been the dominated methods for protecting privacy of individual information. However, those algorithms do not pay full attention to data mining results, which may lead to sensitive rules leakages. While some algorithms are designed for preserving the rule liking with sensitive information, it may degrade the accuracy of other non-sensitive rules. Thus, further investigation, focusing on combining data and rule hiding, may be beneficial, specifically, when taking into account the interactive impact of sensitive and non-sensitive rules, the dependence relationship of the sensitive rules and problem-domain knowledge.

Fifth, various data distortion algorithms have been designed and developed for protecting privacy of individual information. However, those algorithms are usually limited to specialized type of data like integer and float numerical value. Further research could pay more attention to other types of data like character or hybrid type of data.

Finally, a framework for evaluating selected association rule hiding algorithms has been proposed by Bertino et al. [1]. Future research can consider testing the proposed evaluation framework for other privacy preservation algorithms, such as data distortion or cryptography methods. 


\section{Conclusions}

PPDM has recently emerged as a new field of study. A portfolio of algorithms has been suggested for possible solutions. As a new comer, PPDM may offer a wide application prospect but at the same time it also brings us many issues / problems to be answered. In this study, we conduct a comprehensive survey of 29 prior studies to find out the current status of PPDM development. We propose a simplified taxonomy to help understand the problem and explore possible research issues. We also examine the strengths and weaknesses of different privacy preserving techniques and summarize general principles from early research to guide the selection of PPDM algorithms. As part of future work, we plan to formally test a complete spectrum of PPDM algorithms.

\section{References}

1. Bertino, E., Fovino, I., and Provenza, L.: A Framework for Evaluating Privacy Preserving Data Mining Algorithms. Data Mining and Knowledge Discovery 11: 2 (September 2005) 121-154

2. Du, W., and Zhan, Z.: Building Decision Tree Classifier on Private Data. In: Proc. Of the IEEE ICDM Workshop on Privacy, Security and Data Mining (PSDM'02), Maebashi City, Japan ( Dec. 2002) 1-8

3. Du, W., and Zhan, Z.: Using Randomized Response Techniques for Privacy- Preserving Data Mining. In: Proc. of the Ninth ACM SIGKDD Int. Conf. on Knowledge Discovery and Data Mining, Washington, D.C. (Aug. 2003)

4. Evfimievski, A., Gehrke, J., and Srikant, R.: Limiting Privacy Breaches in Privacy Preserving Data Mining. In: Proc. of the Twenty-second ACM SIGMODSIGACTSIGART Symposium on Principles of Database Systems, San Diego, California (June 2003) 211-222

5. Evfimievski, A., Srikant, R., Agarwal, R., and Gehrke, J.: Privacy Preserving Mining of Association Rules. In: Proc. of the 8th ACM SIGKDD Int. Conf. on Knowledge Discovery in Databases and Data Mining (KDD’02), Edmonton, Alberta, Canada (July 2004) 217-228

6. Islam, M. Z., and Brankovic, L.: A Framework for Privacy Preserving Classification in Data Mining. In: Proc. of the 2nd workshop on Australasian information security, Data Mining and Web Intelligence, and Software Internationalization (AISW'04, AWDM\&WI'04, AWSI'04), Dunedin, New Zealand (Jan. 2004) 163-168

7. Kantarcioglu, M., and Clifton, C.: Privacy-preserving Distributed Mining of Association Rules on Horizontally Partitioned Data. In: Proc. of ACM SIGMOD Workshop on Research Issues in Data Mining and Knowledge Discovery (DMKD) (June 2002)

8. Kantarcioglu, M., and Clifton, C.: Assuring Privacy when Big Brother is Watching. In: Proc. of the 8th ACM SIGMOD Workshop on Research Issues in Data Mining and Knowledge Discovery, Privacy \& Security (2003) 88-93

9. Klusch, M., Lodi, S., and Moro, G.: Distributed Clustering Based on Sampling Local Density Estimates. In: Proc. of the 18th Int. Joint Conf. on Artificial Intelligence (IJCAI'03), Acapulco, Mexico (Aug. 2003) 485-490

10. Lindell, Y., and Pinkas, B.: Privacy Preserving Data Mining. In: Advances in Cryptology CRYPTO 2000, Springer-Verlag (Aug. 2000) 36-54 
11. Merugu, S., and Ghosh, J.: Privacy-Preserving Distributed Clustering Using Generative Models. In: Proc. of the 3rd IEEE Int. Conf. on Data Mining (ICDM'03), Melbourne, FL, USA (Nov. 2003) 211-219

12. Meyerson, A. and Williamsy, R.: On the Complexity of Optimal K-Anonymity. In: Deutsch A, ed. Proc. of the 23rd ACM SIGACT- SIGMOD-SIGART Symposium on Principles of Database Systems (PODS 2004). New York: ACM (2004) 223-228

13. Natwichai1, J., Li, X., and Orlowska, M.: Hiding Classification Rules for Data Sharing With Privacy Preservation. In: Proc. of 7th International Conference on Data Warehousing and Knowledge Discovery (DaWaK'05), Copenhagen, Denmark (Aug. 2005) 468-477

14. Oliveira, S. R. M., and Zaïane, O. R.: Privacy Preserving Frequent Itemset Mining. In: Proc. of the IEEE international conference on Privacy, Security and Data Mining (PSDM'02), Maebashi City, Japan (Dec. 2002) 43-54

15. Oliveira, S. R. M., and Zaïane, O. R.: Algorithms for Balancing Privacy and Knowledge Discovery in Association Rule Mining. In: Pro. of the 7th Int. Database Engineering and Applications Symposium (IDEAS'03), Hong Kong, China (July 2003) 54-65

16. Oliveira, S. R. M., and Zaïane, O. R.: Protecting Sensitive Knowledge By Data Sanitization. In: Proc. of the 3rd IEEE Int. Conf. on Data Mining (ICDM'03), Melbourne, Florida, USA (Nov. 2003b) 613-616

17. Oliveira and, S. R. M., and Zaïane, O. R.: Privacy Preserving Clustering by Data Transformation. In: Pro. of the 18th Brazilian Symposium on Databases, Manaus, Amazonas, Brazil (Oct. 2003c) 304-318

18. Oliveira, S. R. M., and Zaïane, O. R.: Achieving Privacy Preservation When Sharing Data for Clustering. In: Pro. of the Int. Workshop on Secure Data Management in a Connected World (SDM'04), In Conjunction with the 30th Very Large Data Base Conference (VLDB'04), Toronto, Canada (Aug. 2004a) 76-82

19. Oliveira, S. R. M., Zaïane, O. R., and Saygin, Y.: Secure Association Rule Sharing. In: PAKDD 2004b 74-85

20. Rizvi, J., and Haritsa, R.: Maintaining Data Privacy in Association Rule Mining. In: Pro. of the 28th Very Large Data Base Conf. (VLDB'02), Hong Kong, China (Aug. 2002) 682-693

21. Saygin, Y., Verykios, V., and Clifton, C.: Using Un-knowns to Prevent Discovery of Association Rules. ACM SIGMOD Record 30: 4 (2001)

22. Saygin, Y., Verykios, V., and Elmagarmid, A.: Privacy Preserving Association Rule Mining. In: Proc. of 12th Int. Workshop on Research Issues in Data Engineering (RIDE) (Feb. 2002).

23. Vaidya, J., and Clifton, C.: Privacy Preserving Association Rule Mining in Vertically Partitioned Data. In: Proc. of the 8th ACM SIGKDD Int. Conf. on Knowledge Discovery and Data Mining (2002) 639-644

24. Vaidya, J., and Clifton, C.: Privacy-Preserving K-Means Clustering over Vertically Partitioned Data. In: Proc. of the 9th ACM SIGKDD Int. Conf. on Knowledge Discovery in Data (KDD’03), Washington D.C., USA (Aug. 2003) 206-215

25. Vaidya, J., and Clifton, C.: Privacy-Preserving Decision Trees over Vertically Partitioned Data. In: Proc. of the 19th Annual IFIP WG 11.3 Working Conf. on Data and Applications Security (DAS'05), Storrs, CT, USA (Aug. 2005) 139-152

26. Veloso, A., Meira, Jr., W., Parthasarathy, S., and de Carvalho, M.: Efficient, Accurate and Privacy-Preserving Data Mining for Frequent Itemsets in Distributed Databases. In: Proc. of the 18th Brazilian Symposium on Databases, Manaus, Amazonas, Brazil (Oct. 2003) 281-292 
27. Verykios, S., Elmagarmid, K., Elisa, B., Saygin, Y. and Elena, D.: Association Rule Hiding. IEEE Transactions on Knowledge and Data Engineering (2003)

28. Verykios, S., Bertino, E., Fovino, I., Provenza, L., Saygin, Y., and Theodoridis, Y.: Stateof-the-art in Privacy Preserving Data Mining. ACM SIGMOD Record 33: 1 (March 2004) 50-57

29. Wang, K., Yu, S., and Chakraborty, S.: Bottom-Up Generalization: A Data Mining Solution to Privacy Protection. In: Proc. the 4th IEEE Int. Conf. on Data Mining (ICDM'04), Brighton, United Kingdom (Nov. 2004) 249-256

30. Wenliang, D. and Zhijun, Z.: A Study of Several Practical Approach to Solve Secure Multiparty Computation Problems. In: Pro. of the Int. Conf. on Computer Networks and Mobile Computing (ICCNMC'03) (2003)

31. Xia, Y., Yang, Y., Chi, Y., and Muntz, R. R.: Mining Association Rules with Nonuniform Privacy Concerns. Technical Report CSD-TR No. 040015, University of California (March 2004)

32. Yang, Z., Zhong, S., Wright, R. N.: GrC. Privacy-Preserving Model Selection. In: Proc. of the IEEE Int. Conf. on Granular Computing (2006)

33. Zhan, J. Z., Matwin, S., and Chang, L.: Privacy-preserving Collaborative Association Rule Mining. DBSec (2005) 153-165 